\title{
Design and Implementation of PLC-Based Automatic Sun tracking System for Parabolic Trough Solar Concentrator
}

\author{
Jinping Wang ${ }^{1,2}$, Jun Zhang ${ }^{2}$,Yanfeng $\mathrm{Cui}^{2}$ and Xiaolong $\mathrm{Bi}^{2}$ \\ ${ }^{1}$ College of Energy \& Environment Engineering, Southeast University EDP Sciences, Editorial Department, 211167, China \\ 2 School of Energy \& Power Engineering, Nanjing Institute of Technology, 210096, China
}

\begin{abstract}
A sun-tracking system for parabolic trough solar concentrators (PTCs) is a control system used to orient the concentrator toward the sun always, so that the maximum energy can be collected. The work presented here is a design and development of PLC based sun tracking control system for PTC. Sun tracking control system consists of a Programmable Logic Controller (PLC) and a single axis hydraulic drives tracking control system. Hydraulic drives and the necessary tracking angle algorithm have been designed and developed to perform the technical tasks. A PLC unit was employed to control and monitor the mechanical movement of the PTC and to collect and store data related to the tracking angle of PTC. It is found that the tracking error of the system is less than $0.6^{\circ}$. Field experience shows that tracking algorithm act stable and reliable and suit for PTCs.
\end{abstract}

\section{Introduction}

A parabolic trough is a type of solar thermal collector that is straight in one dimension and curved as aparabola in the other two, lined with a polished metal mirror [1]-[3]. The aperture angle in parabolic trough solar collectors (PTC) is the angle between the axis of the parabola and the line connecting the focus on one end of that parabola [4]. A receiver tube with a fluid flowing inside that absorbs concentrated solar energy from the tube walls and raises its enthalpy is installed in this focal line. The collector is provided with one-axis solar tracking to ensure that the solar beam falls parallel to its axis. When a collector (its aperture normal) is not pointing directly at the Sun, some of the energy that could be collected is being lost [5]. Sun-tracking systems are designed in a way to track the sun on a single axis or in a way to track the sun on two axes. Over the years, several researchers have studied the solar tracking systems with different modes and electromechanical module to improve the efficiency of solar systems. Soteris A. Kalogirou uses three Light-Dependent Resistors (LDRs) as sensors to detects whether the collector is focused [6]. Dhanabal R, Bharathi $\mathrm{V}$ et al.make comparison of efficiencies of suntracking systems with static panel single-axis tracking system and dual-axis tracking system with fixed mount [7]. Ali Al-Mohamad presents a Sun-tracking design, whereby the movement of a photo-voltaic module was controlled to follow the Sun's radiation using a Programmable Logic-Controller (PLC) unit. All electronic circuits and the necessary software have been designed and developed to perform the technical tasks. A
PLC unit was employed to control and monitor the mechanical movement of the PV module and to collect and store data related to the sun's radiation [8]. Chong $\mathrm{K}$ $\mathrm{K}$, Tan $\mathrm{M} \mathrm{H}$ use two sun-tracking methods for the heliostat, which are conventional Azimuth-Elevation and revolutionary Spinning-Elevation methods [9]. However, for parabolic trough solar collectors (PTCs), it can only use direct solar radiation, called Direct Normal Irradiance (DNI). PTCs are designed to operate with tracking about only one axis [10]. A good sun-tracking system must be reliable and able to track the sun at the right angle even in the periods of cloud days. Although the degree of accuracy required to depend on the specific characteristics of the solar concentrating system being analyzed, generally the higher the system concentration the higher the tracking accuracy will be needed [11].

In this paper, a PLC-based sun-tracking system for parabolic trough solar concentrator which could track the sun along one axes was designed and implemented. In the system, the tracking angle was calculated in real time based on solar position by the PLC controller. The PLC program was administered according to these calculated tracking angle, and by controlling the hydraulic drives of the parabolic trough solar collector with the rotary encoder signal received from the input module of the PLC, it was ensured that the PTC moves along one-axe, and maximum benefit was derived from solar energy by providing that the PTCs system are oriented at a right angle to the sun.

\section{Design of the PLC-Based sun-tracking for parabolic trough solar concentrator}




\subsection{Overview of PLC-Based sun-tracking}

The overall structure of PLC-based automatic suntracking system is shown in Fig. 1. The PLC based suntracking has the following components: PLC control system and field equipment. The difference between angle sensor to measure the PTC position and angle by PLC calculated is calculated. If this difference is greater than the threshold set in the program execution cycle, the PLC control system will produce hydraulic driven action commands enable parabolic trough solar concentrator running from east to west or west to east and track the sun. The control system by PLC controller system and field equipment constitute a feedback closed-loop control system based a rotary encoder.

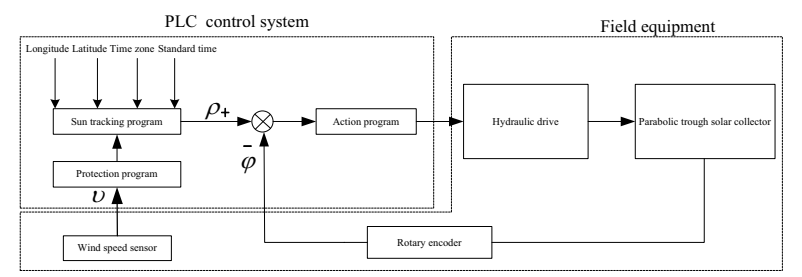

Figure 1. Overall structure of PLC-based automatic suntracking system

\subsection{Tracking angle Algorithm}

Parabolic trough solar collectors are designed to operate with tracking about only one axis. A tracking drive system rotates the collector about an axis of rotation until the sun central ray and the aperture normal area are coplanar. Fig. 2 shows how the rotation of a collector aperture about a tracking axis $r$ [12].

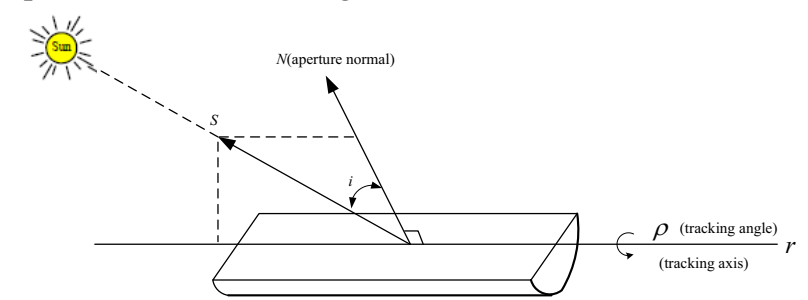

Figure 2.A single-axis tracking aperture

The tracking axis brings the sun ray unit vector $S$ into the plane formed by the aperture normal and the tracking axis. $i$ is the cosine of incidence angle, $\rho$ is the tracking angle. Fig. 3 shows the $u-r-b$ coordinate system, where $r$ is the tracking axis, $b$ is the axis that always remains parallel to the earth surface and $u$ is the third orthogonal axis [13].

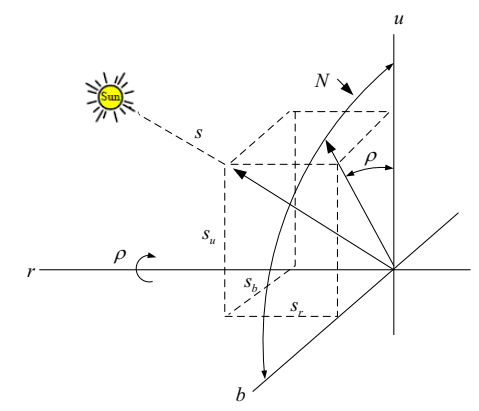

Figure 3. The $u-r-b$ coordinate system
The tracking angle is written as Eq. (1) [12]:

$$
\rho=\tan ^{-1}\left(-\frac{S_{u}}{S_{b}}\right)
$$

The cosine of incidence angle is written as Eq. (2) [12]:

$$
i=\cos ^{-1}\left(\sqrt{S_{b}^{2}+S_{u}^{2}}\right)
$$

where $S_{u}$ is projection of the sun ray unit vector $S$ on the $u$ axis, $S_{b}$ is projection of the sun ray unit vector $S$ on the $b$ axis. Because of the parabolic trough collectors is horizontal tracking axis in general, the tracking angle and cosine of incidence angle can also be written as Eq. (3) [13]:

$$
\rho=\tan ^{-1}\left[\frac{\sin \left(\gamma-\alpha_{s}\right)}{\tan \alpha}\right]
$$

$\alpha_{s}$ is azimuth angle, $\alpha$ is the altitude angle.

\section{Experimental and results}

To test the aforementioned methodology, a prototype of an parabolic trough solar collector withlength of $50 \mathrm{~m}$ and aperture of $5.77 \mathrm{~m}$ has been constructed in the Hengxi town, Jiangning District, Nanjing City, Jiangsu Province, China (located at latitude $31.45^{\circ}$ and longitude $118.46^{\circ}$; see Fig. 4).

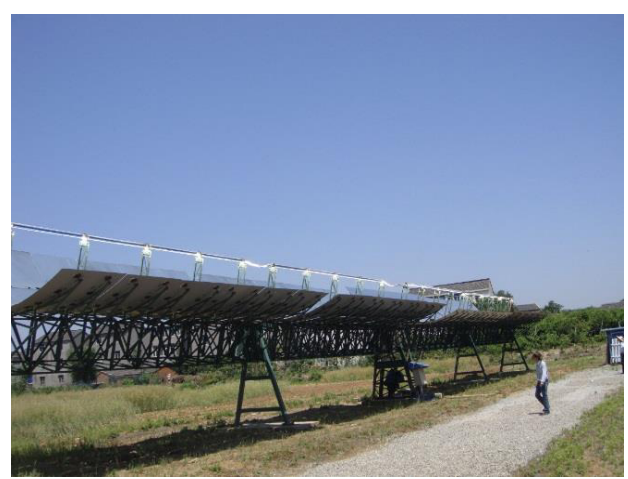

Figure 4. A prototype of parabolic trough solar collector that has been constructed at HengXi town in China

We have selected 4 typical dates to carry out experimental research,these 4 days are: March 20(vernal equinox), June 21(summer solstice), September 23(autumnal equinox), and December 22(winter solstice) in 2014. The solar altitude angle varies throughout the day as shown in Figure 5. The elevation is $0^{\circ}$ at sunrise, reaches maximum value when the sun is directly overhead, then it begin to fall, is down to $0^{\circ}$ at sunset. The solar altitude angle reaches the maximum value of the year in the summer solstice (June 21) at solar noon time, the maximum of solar altitude angle is $81.03^{\circ}$, however, the value is $34.89^{\circ}$ in the winter solstice (December 22) at solar noon time, the difference between the two values is $34.89^{\circ}$. The azimuth angle is the compass direction from which the sunlight is coming. At 
solar noon, the sun is always directly south in the northern hemisphere and directly north in the southern hemisphere. The azimuth angle varies throughout the day as shown in Figure 1,2. The azimuth angle reachs $180^{\circ}$ at solar noon time. The range of azimuth (sunrise to sunset)

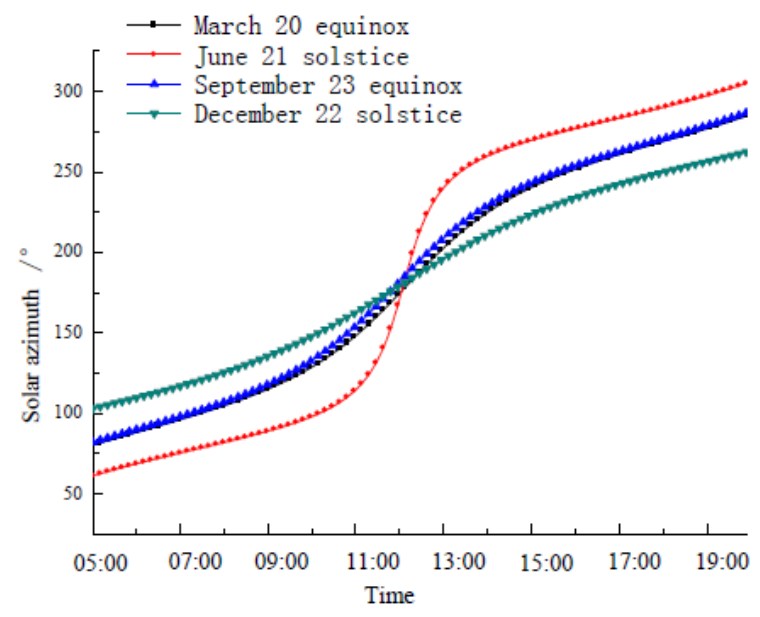

reached the maximum value of the summer solstice (June 21 ), is $236.08^{\circ}$. The value is $124.92^{\circ}$ in the winter solstice (December 22). From the change regulation of the angle of the azimuth angle and the solar altitude angle.

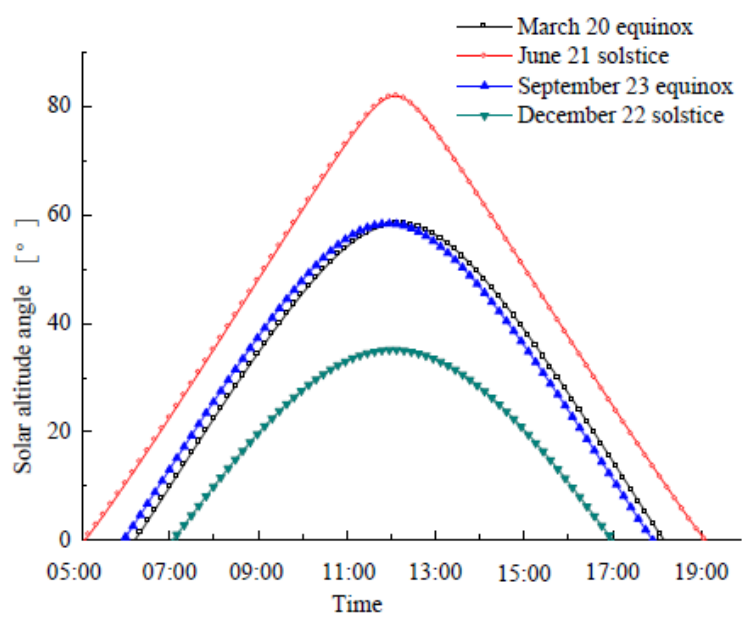

Figure 5. Variation of solar altitude angle And azimuth angle

Fig. 6 is variation of the tracking angle with 4 days in 2014.we can see that the tracking angle range of PTC is run within the range of 0 to $180^{\circ}$.

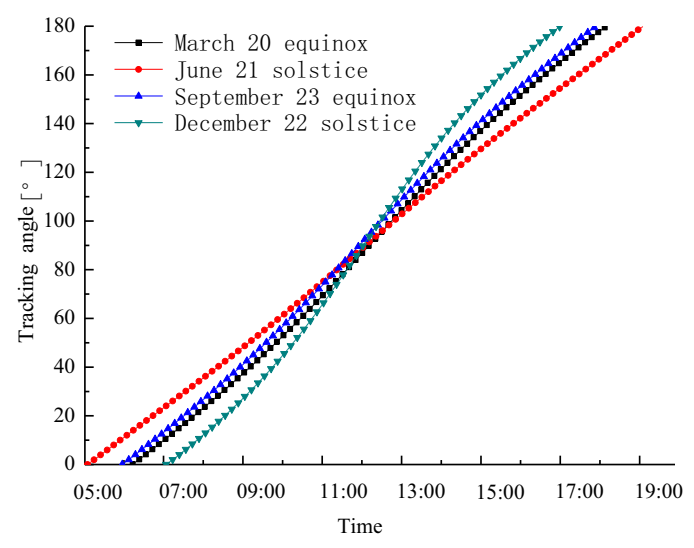

Figure 6. Variation of the tracking angle

The trajectory of the tracking angle is more complex, and the daily movement law is not the same. The range of the rotational trajectory of PTC reaches maximum in the summer solstice, reaches minimum in the winter solstice. Figure.7 is variation of change rate of tracking angle of PTC. We can see that although the tracking angle range of PTC reaches minimum in the winter solstice, but the change rate of tracking angle of PTC is the fastest, is $0.398^{\circ} / \mathrm{min}$ at solar noon. Although the tracking angle range of PTC reaches maximum in the summer solstice, but the change rate of tracking angle of PTC is running slower than other dates, the maximum value of the day is $0.232 \% \mathrm{~min}$, the difference of these two days is $0.166 \%$ min at solar no one in the summer solstice and winter days .

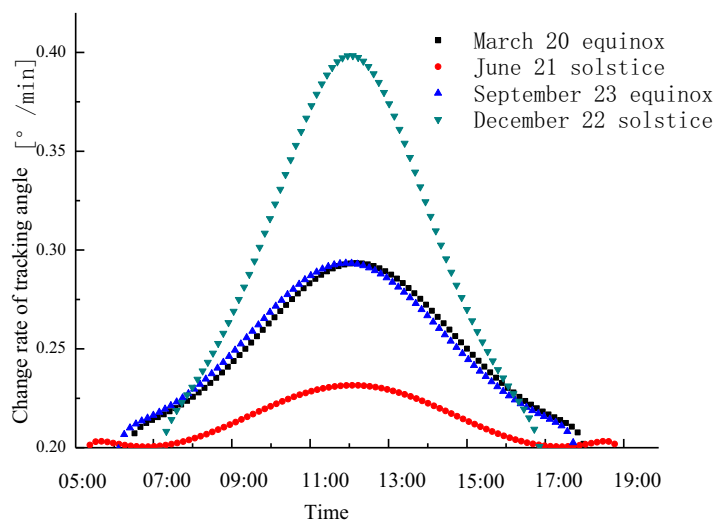

Figure 7. Variation of change rate of tracking angle of PTC

The vernal equinox and autumnal equinox had little difference. The change rate of tracking angle of PTC runs fastest at solar noon in every day .Through the above analysis, we can know the trajectory of the parabolic trough solar concentrator is very complicated, so the suntracking control system for parabolic trough solar collectors needs to be designed according to the nonlinear control system. A procedure for a Solar Position Algorithm (SPA) to calculate the solar zenith and azimuth angle with uncertainties equal to $\pm 0.0003^{\circ}$ in the period from the year -2000 to 6000 in Literature [14]. We compare SPA algorithm with the algorithm designed in this paper. Fig. 8 is the contrast curve of calculated tracking angle by the PLC system, the encoder output angle and the angle by SPA algorithm calculated in 1 hour. The maximum deviation of the angle of the SPA algorithm and the PLC system calculated is $0.12^{\circ}$. The maximum deviation from the angle of the encoder output angle and the PLC system calculated is $0.4^{\circ}$. Therefore, the tracking error of the system is less than $0.6^{\circ}$. 


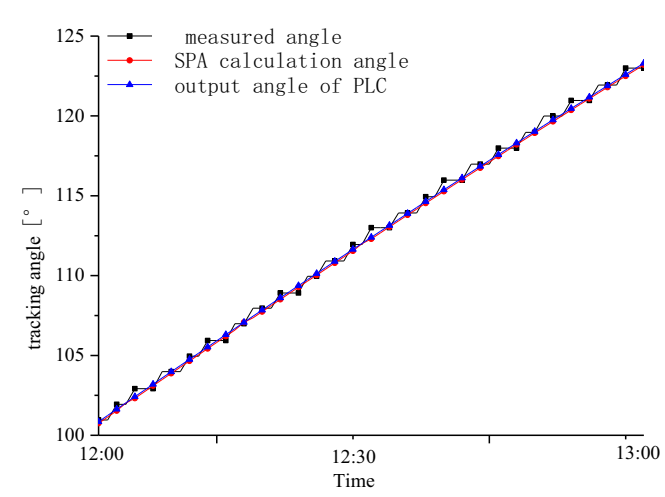

Figure 8. Contrast change curve with different angles

\section{Conclusions}

In this research work design of sun tracking system for parabolic trough solar concentrator has been considered by using PLC and hydraulic drive. Using this automatic sun-tracking system can make the parabolic trough solar concentrator automatic, reliable and does not depend on the weather when tracking the sun. The tracking angle algorithm presented in this paper has a high degree of accuracy,experimental results show that the tracking error of the system is less than $0.6^{\circ}$. The sun tracking system developed in this study provides easy installation, simple mechanism and less maintenance.

\section{Acknowledgments}

This study was simultaneously supported by Key Laboratory Open Fund Project of Jiangsu Provincial Key Laboratory of Solar Energy Science and Technology, the Natural Science Foundation of the Jiangsu Higher Education Institutions of China(Grant No.14KJD470003) and Youth Fund Key Project of Nanjing Institute of Technology(Grant No.QKJA201402).

\section{References}

1. H. Lund. Renewable energy strategies for sustainable development[J]. Energy, 32(6):912-919, (2007).

2. F. Chen, N. Duic, L. M. Alves, et al. Renewislands-Renewable energy solutions for islands[J]. Renewable \& Sustainable Energy Reviews, 11(8):1888-1902, (2007).

3. J. P. Wang, J. Wang, X. L. Bi, and X. Wang,
"Performance Simulation Comparison for Parabolic Trough Solar Collectors in China," International Journal of Photoenergy, vol. 2016, Article ID 9260943, 16 pages, (2016).

4. W. Jinping, W. Jun, Z. Yaoming, and B. Xiaolong, "Analysis of heat transfer characteristics for parabolic trough solar collector," Transactions of the Chinese Society of Agricultural Engineering, 31, no. 7, pp. 185-192, (2015) (Chinese).

5. E. Zarza, L. Valenzuela, J. León, et al. Direct steam generation in parabolic troughs: Final results and conclusions of the DISS project [J]. Energy, 29, pp. 635-644, (2004).

6. S. A. Kalogirou. Design and construction of a oneaxis sun-tracking system[J]. Solar Energy, 57(6), pp. 465-469, (1996).

7. R. Dhanabal, V. Bharathi, R. Ranjitha, et al. Comparison of Efficiencies of Solar Tracker systems with static panel Single-Axis Tracking System and Dual-Axis Tracking System with Fixed Mount[J]. International Journal of Engineering \& Technology, 5, pp. 0975-4024, (2013).

8. A. Al-Mohamad. Efficiency improvements of photovoltaic panels using a Sun-tracking system [J]. Applied Energy, 79, pp. 345-354, (2004).

9. K. K. Chong, M. H. Tan. Comparison Study of Two Different Sun-Tracking Methods in Optical Efficiency of Heliostat Field [J]. International Journal of Photoenergy, pp. 483-490, (2012).

10. M. Ozturk. Exergy Analysis of Low and High Temperature Water Gas Shift Reactor with Parabolic Concentrating Collector [J]. Clean Energy Systems and Experiences. pp. 107-130, (2010).

11. K. K. Chong, and C. W. Wong. General formula for on-axis sun-tracking system and its application in improving tracking accuracy of solar collector [J]. Solar Energy, 83, pp. 298-305, (2009).

12. W. B. Stine, R. W. Harrigan. Solar energy fundamentals and design: with computer applications [M]. Wiley, (1985).

13. R. V. Padilla. Simplified Methodology for Designing Parabolic Trough Solar Power Plants [D]. Dissertations \& Theses - Gradworks, (2011).

14. I. Reda, A. Andreas, I. Reda, et al. Solar position algorithm for solar radiation applications [J]. Solar Energy, 76, pp. 577-589, (2004). 